\author{
Mediterranean BioMedical Journals \\ Integrative Journal of Medical Sciences \\ 2020, Volume 7, ID 125 \\ DOI: 10.15342/ijms.7.125
}

RESEARCH ARTICLE

\title{
Evaluation of the Interpretation Criteria of Western Blot Profiles for the HIV-1 Infection Diagnosis in a South Moroccan Group
}

\author{
Smail CHADLI ${ }^{1}$ (iD), Hasna AIT SAID ${ }^{2}$, Fatima Zahra FADIL ${ }^{1}$, Fatima BENLMELIANI ${ }^{2}$, \\ Jamila SARDI $^{2}$, Samira KHARBOUCH ${ }^{2}$, Nadia HASSAINI ${ }^{2}$, Maryem EL BASSBASSI ${ }^{2}$, Naima TAQARORT ${ }^{3}$, \\ Said OULKHEIR ${ }^{1}$, Mohamed AGHROUCH ${ }^{2}$
}

\begin{abstract}
1 Higher Institute of Nursing Professions and Health Techniques, ISPITS Agadir, Morocco.
2 Laboratory of Medical Analysis, Hospital Hassan II, Agadir, Morocco

3 Polydisciplinary faculty of Taroudant, Ibn Zohr University, Agadir.
\end{abstract}

\begin{abstract}
Introduction : Western Blot (WB) is the most commonly used method to confirm the presence of antibodies against Human Immunodeficiency Virus type 1 (HIV-1). The interpretation of WB, however, poses difficulties since of the multiplicity of criteria proposed by various international organizations. Some criteria generate an unacceptable number of indeterminate results, thus preventing the early management of patients. The present work aims to evaluate the different criteria for interpretation of WB according to various organizations compared to viral load test.

Material and methods : This is a study carried out on a total of 2618 Western Blot profiles registered during a period of 56 month (from January 2013 to September 2017) at the Laboratory of Medical Analysis of Regional Hospital Center in Agadir. Results : The results obtained indicate that some organizations have very interesting values concerning the positivity of the WB profiles; WHO (94.84\%), CDC/ ASTPHLD (94.61\%) and SFTS (93.09\%). For the negative profiles, all the organizations have identical values with $3.59 \%$. Regarding indeterminate profiles, the WHO gave the minimum of these cases (1.57\%) followed by CDC / ASTPHLD (1.8\%) and SFTS (3.32\%). The WHO provided very interesting values for sensitivity (98.93\%), specificity (91.26\%), positive predictive value (99.64\%) and negative predictive value (74.60\%). Similarly, the CDC / ASTPHLD criteria remain favorable and are highly consistent with those of WHO (p = 0.8113).

Conclusion : Sensitivity, specificity, and predictive values are characteristics of WB test that interpret the results and allow applying them to patients. In our context, it is recommended to adopt the WHO criteria to facilitate the interpretation of western blot profiles. The CDC / ASTPHLD criteria remain favorable to adoption.
\end{abstract}

KEYWORDS : HIV-1; Western Blot Method; interpretation criteria; indeterminate profiles.

Correspondence : Pr. Smail Chadli, Higher Institute of Nursing Professions and Health Techniques, ISPITS Agadir, Morocco ; Phone : +212663112099 ; Email : schadli@gmail.com

Copyright (c) 2020 Smail Chadli et al.

This is an open access article distributed under the Creative Commons Attribution 4.0 International, which permits unrestricted use, distribution, and reproduction in any medium, provided the original work is properly cited.

\section{INTRODUCTION}

The Human Immunodeficiency Virus (HIV) infection is a major global public health problem. The latest estimates point to 36.7 million people living with HIV worldwide, with $70 \%$ of them in Africa. In 2016, 1.8 million new HIV infections and 1.0 million aids-related deaths were identified [1]. The burden of the AIDS epidemic continues to vary considerably across countries and regions. It becomes necessary to make a certainty, reliability and accuracy diagnosis whose main objective is to minimize the socioeconomic consequences 
threatening public health. In Morocco, HIV prevalence remains low in the general population $(0.1 \%)$. At the end of 2016, the number of people living with HIV is estimated at 22.000, with 1.000 new infections and 700 deaths a year [2]. Three regions of Morocco, including the Souss Massa region, alone account for $56 \%$ of reported HIV / AIDS cases [3-4].

The serological diagnosis of HIV infection in Morocco is based on a screening technique, followed by a very specific confirmatory test regularly a Western blot test (WB) [5]. Although the overall sensitivity and the specificity of the Western blot test are high, its interpretation, however, poses difficulties since of the multiplicity of criteria proposed by various international organizations. Some criteria generate an unacceptable number of indeterminate results, thus preventing the early management of patients. In fact, the indeterminate profiles (which do not satisfy the criteria of positivity or negativity), obtained after interpretation according to the criteria adopted by an organization, require a second test to be carried out in three or four weeks next. This is not appropriate in routine hospital practice. It is often very challenging to announce such results to patients. Additionally, it can cause a delay of early management of patients or sometimes generate lost sight target.

The present work proposes to evaluate the criteria for the interpretation of western blot profiles for the HIV-1 infection diagnosis used in Moroccan laboratory conditions according to various organizations, with the aim of deducing the one that gives the minimum of indeterminate profile and the maximum of reliability and performance cases.

\section{MATERIALS AND METHODS}

This is a study carried out on a total of 2618 Western Blot profiles (HIV BLOT 2.2 de MP Diagnostics) registered during a period of 56 month (from January 2013 to September 2017) at the Laboratory of Medical Analysis of Regional Hospital Center, Hassan II Hospital in Agadir. This study is based on subjects declared positive or doubtful on a screening test (the enzymelinked immunosorbent assay "ELISA" or rapid test). The WB was completed and the profiles were interpreted according to the criteria approved by the seven international organizations: WHO (World Health Organization) [6], CDC (Center for Disease Control) [7], ARC (American Red Cross) [8], SFTS (French Blood Transfusion Society) [9], CRSS (Consortium for the Standardization of Retrovirus Serology) [10], FDA (Food and Drug Administration) [11] and ASTPHLD (Association of States and Public Health Diseases) [12]. A PCR test (Abbot Real Time HIV-1, Applied Biosystems) was set up allowing the resolution of the status of patients with undetermined WB profiles. All the indeterminate profiles of the interpretation obtained by each organization were compared with those noted by the viral load test. This was used as a reference test, in order to decide between positivity and negativity of indeterminate profiles.

All statistical tests were performed. The criteria: sensitivity, specificity, positive predictive value and negative predictive value were obtained for each organization. The result is considered statistically significant at a value of $\mathrm{p}<0.05$.

\section{RESULTS}

The present study is based on 2618 subjects declared positive or suspicious on a screening test. The serum samples were analyzed by the Western blot test and profiles were interpreted according to the seven organizations criteria. The organizations WHO, CDC / ASTPHLD and SFTS had interesting percentages concerning the revelation of the positivity of the WB profiles. They were respectively $94.84 \%, 94.61 \%$ and 93.09\%. The CRSS, ARC, and FDA reported positivity percentages respectively $92.2 \%, 83.54 \%$, and $80.6 \%$. All organizations had an identical portion of negative WB profiles (3.59\%). Regarding indeterminate profiles, WHO presented the minimum of these cases (1.57\%), followed by CDC / ASTPHLD (1.8\%) and SFTS (3.32\%). 4.13\%, $12.87 \%$ and $15.81 \%$ were the portions of indeterminate profiles exposed respectively by CRSS, ARC and FDA. The comparison of the distribution of WB profiles (positive, negative and undetermined) versus to the profiles of the viral load tests (positive and negative) showed, for all the different organizations, very highly significant differences ( $p<0.0001)$. The comparison of interpretation results of WB profiles between the different organizations (two by two), revealed the existence of a high concordance between the criteria approved by WHO and CDC / ASTPHLD ( $=0.8113$ ). However, there was a highly significant difference between the other results of the organizations compared $(\mathrm{p}<0.0001)$.

The different interpretation criteria of Western blot provide different degrees of sensitivity and specificity (Table 1). The highest sensitivity was found for the WHO (98.73\%) and CDC / ASTPHLD (98.49\%) criteria. The sensitivity of the WB test by other organisms was in descending order: SFTS (97.01\%), CRSS (96.14\%), ARC (87.41\%) and FDA $(84.30 \%)$. It should be noted that WHO and CDC / ASTPHLD also had very interesting values for specificity (91.26\%) and positive predictive value (PPV) (99.64\%). In addition, the WHO had the largest negative predictive value (NPV), with a percentage of $74.6 \%$. However, the NPV found for the FDA criteria was mainly the lowest among the seven organisms (19.30\%).

\section{DISCUSSION}

Western Blot is the most commonly used test for confirming HIV serologic diagnosis. Correct interpretation of WB results is decisive, but, the test still suffers from a lack of consensus on the interpretation criteria adopted by various organizations. The results of interpreting WB profiles change from one organization to another. Indeed, in this study, four of the seven selected organizations criteria had converged values for the positivity of WB profiles. These are WHO, CDC/ASTPHLD and SFTS, with scores of $94.84 \%$, $94.61 \%$ and $93.09 \%$ respectively. These proportions of the positive profiles are higher compared to those obtained in India by these four criteria (an identical score of $69.6 \%$ ) [13]. However, for these authors, the FDA has the most interesting value $(70.5 \%)$ regarding the positivity of WB profiles, while in our case it was the lowest (80.6\%). As regards the frequency of indeterminate profiles in our sample, the WHO gave the minimal percentage $(1.57 \%)$, while the high proportions of these cases are attributed to the FDA and ARC criteria respectively $15.81 \%$ and $12.87 \%$. In a work done in Ethiopia, it was reported that the lowest frequency of these indeterminate profiles is obtained by the ARC (0.8\%) [14]. Circulating HIV-1 strains have shown that in Morocco there is a predominance of subtype B [15-16], while in India and in Ethiopia subtype $\mathrm{C}$ is predominant $[13,17]$. These two sub-types diverge from each other by an average of $25 \%$ in the pol gene and $15 \%$ in the gag 
gene. The variability of HIV strains and the qualities of each commercial HIV test kit could explain the frequencies of different positive or indeterminate profiles from one region to another [18]. Criteria of Western Blot should always be evaluated in a specific geographic area, with sera collected locally, to determine susceptibility and specificity values. Indeed, the results of the tests can be attributed also to the specificity of the viral proteins adopted as criteria for each organization. Our results show, in particular, the flexibility of WHO positivity criteria, which are based on the requirement of only two of the three envelope-specific bands. In contrast, the criteria approved by FDA require the existence of p31 and p24 proteins. While the ARC criteria, require at least one band of each of the three genes (env, pol and gag), and these are the cases that are not always possible. In this regard, a study carried out in Morocco [5] reported that the inclusion of gag and/or pol proteins immunoreactivity in the WB test positivity criteria could increase the cases of indeterminate profiles. This could jeopardize the diagnosis confirmation of HIV infection in the absence of these proteins, especially in the early stage and advanced stages of infection.

A PCR test was set up allowing the resolution of the status of patients with undetermined WB profiles. The distribution of patient profiles on the basis of viral load, compared with that of each organization, showed that there is a very large statistically significant difference. It is crucial in this situation to evaluate the performance of the criteria organization, thus making it possible to determine their accuracy, and subsequently to choose the best ones to adopt for interpretation in the Moroccan context. In this regard, our study found that the highest sensitivity was found for the WHO criteria (98.73\%) and the lowest sensitivity with FDA (84.30\%). Regarding specificity, the WHO and CDC / ASTPHLD have a very interesting value with a percentage of $91.26 \%$, while the ARC criteria remain the least specific $(81.03 \%)$. The present study also revealed that WHO has very interesting values for the positive and negative predictive value, which are respectively $99.64 \%$ and $74.6 \%$. However, FDA represents the organism that gave very low value (NPV $=19.30 \%)$. The criteria adopted by the WHO then gave the best results in our population than the other criteria. Comparing the results of WHO with those of other organizations, it was found that there is no statistically significant difference between the WHO and

\section{REFERENCES}

[1] Joint United Nations, Programme on HIV/AIDS (2017). UNAIDS Data 2017. Geneva, Switzerland. (http://www.unaids.org/sites/default/files/media_asset/201 70720_Data_book_2017_en.pdf).

[2] Ministère de la santé avec l'appui de Fonds Mondial (2017). JM Sida 2017, réalisations et perspectives. Maroc. http://www.sante.gov.ma/Documents/2017/11/JMS\%2020 17\%20BROCHURE\%20fr.pdf

[3] Ministère de la santé. Plan stratégique national de lutte contre le sida; 2012-2016, Maroc.

[4] Chadli S., Aghrouch M., Taqarort N., Malmoussi M., Ouagari Z., Moustaoui F., Bourouache M., Oulkheir S. (2018). Neuromeningeal cryptococcosis in patients infected with HIV at Agadir regional hospital, (SoussMassa, Morocco). Journal of Medical Mycology, 28 (1), 161-116. https://doi.org/10.1016/j.mycmed.2017.10.006.

[5] Admou B., Zougaghi L., Soraa N. et al. (2009). HIV-1 western-blot patterns and clinical stages in a Moroccan population. Revue Francophone des Laboratoires, 2009 (416): 19-22. https://doi.org/10.1016/S1773035X(09)70270-9
CDC / ASTPHLD criteria. In a study in Taiwan [19], it was suggested that WHO criteria be adopted to facilitate the interpretation of western blot profiles.

In another work done in Iran [20], it was recommended that the ASTPHLD / CDC interpretation criteria be used to interpret the results of $\mathrm{WB}$, since they make it possible to considerably reduce the number of indeterminate interpretations. In the study conducted in Ethiopia [14], it was revealed that the ARC criteria best met the objectives specified for the diagnosis. This suggested that new criteria could be developed to improve WB interpretation in the African context, where the epidemic is most prevalent.

\section{CONCLUSIONS}

Criteria of Western Blot should always be evaluated in a specific geographic area, with sera collected locally, to determine susceptibility and specificity values. Each laboratory must adopt the criteria that best rejoin the objectives defined for the diagnosis. It is essential to mention that organization that require the presence of several proteins as criteria of positivity, give an unacceptable number of indeterminate cases. Sensitivity, specificity, positive predictive value and negative predictive value are characteristics of WB test that interpret the results and allow applying them to patients. In the Moroccan context, it is recommended to adopt the WHO criteria to facilitate the interpretation of western blot profiles. The CDC / ASTPHLD criteria remain favorable to adoption. However, the new criteria could be developed to improve WB interpretation in the African situation.

\section{AUTHORS' CONTRIBUTIONS}

The participation of each author corresponds to the criteria of authorship and contributorship emphasized in the Recommendations for the Conduct, Reporting, Editing, and Publication of Scholarly work in Medical Journals of the International Committee of Medical Journal Editors. Indeed, all the authors have actively participated in the redaction, the revision of the manuscript and provided approval for this final revised version.

\section{COMPETING INTERESTS}

The authors declare no competing interests.

[6] World Health Origanisation (1991). AIDS: proposed criteria for interpreting Western Blot assays for HIV-1, HIV-2, and HTLV 1/HTV-II. Bulletin of the WHO 69 (1): 127-130.

[7] Centers for Disease Control (1989). Interpretation and Use of the Western Blot Assay for Serodiagnosis of Human Immunodesficiency Virus Type 1 Infection. MMWR, 38 (S-7), 1-7.

[8] Sandler SG, Dodd RY, Fang CT. (1988). Diagnostic tests for HIV infection: Serology. In: DeVita VT, Hellman S, Rosenberg SA, eds. AIDS: Etiology, Treatment, and Prevention, Second Edition. Philadelphia: J.B. Lippincott, 121-126.

[9] Koeck J.-L., Dubrous P., Coulot P., Blanchard de Vaucouleurs A. (1997). Problèmes posés par l'interprétation des tests western-blot VIH-1 en 1997. Revue Française des Laboratoires 1997 (294): 69-73. https://doi.org/10.1016/S0338-9898(97)80198-9

[10] McKenna, S.J. (1989). Serological diagnosis of human immunodeficiency virus infection by Western blot testing: The Consortium for Retrovirus Serology Standardization. 
Carlson JR. JAMA 260: 674. Journal of Oral and $\begin{array}{llll}\text { Maxillofacial } & \text { Surgery } 47 \quad \text { (4): } 429-430 .\end{array}$ https://doi.org/10.1016/0278-2391(89)90360-1

[11] Du Pont Diagnostics Company (1987). Human immunodeficiency virus (HIV): Biotech/Du Pont HIV western blot kit for detection of antibodies to HIV. Wilmington, Delaware.

[12] Hausler W. J., Jr. (1988). Report of the Third Consensus Conference on HIV Testing Sponsored by the Association of State and Territorial Public Health Laboratory Directors. Infection control and hospital epidemiology 9: 345-349.

[13] Syed Iqbal H., Balakrishnan P., Sunil S. et al. (2005). HIV-1 Western Blot Assay: What determines an indeterminate status? Indian journal of medical sciences, 59 (10): 443-450.

[14] Meles, H., Wolday, D., Fontanet, A., et al. (2002). Indeterminate Human Immunodeficiency Virus Western Blot Profiles in Ethiopians with Discordant ScreeningAssay Results. Clinical and Diagnostic Laboratory Immunology, 9(1), 160-163. http://doi.org/10.1128/CDLI.9.1.160-163.2002.
[15] Kouyoumjian, S. P., Mumtaz, G. R., Hilmi, N., Zidouh, A., et al. (2013). The epidemiology of HIV infection in Morocco: systematic review and data synthesis. International Journal of STD \& AIDS, 24(7), 507-516. http://doi.org/10.1177/0956462413477971

[16] El Harti E., El Aouad R., Amzazi S., et al. (1997). HIV-1 diversity in Morocco. Aids, 11, 1781-1782

[17] Lihana R. W., Ssemwanga D., Abimiku A., and Ndembi N., (2012). Update on HIV-1 diversity in Africa: a decade in review, AIDS Review, 14 (2), 83-100.

[18] Ndjoyi-Mbiguino A., Bélec L. (2005). Évaluation de trousses de dépistage de l'infection à VIH au Gabon. Cahiers d'études et de recherches francophones / Santé, 15 (1), 23-29.

[19] Weng K.-C., Ho S.-Y., Chang S.-Y. (2015). Clinical experiences in interpretation of HIV-1 Western blot indeterminate results. Journal of Microbiology, Immunology and Infection 48 (2), S134. https://doi.org/10.1016/j.jmii.2015.02.471

[20] Ravanshad, M., Mahboudi F., Sabahi F., Bayanolhagh S. (2006). Indeterminate human immunodeficiency virus western blot results in Iranian patients with discordant screening assay results. Saudi Med. J., 27(8), 1130-1133. 